\title{
EXPLORING THE BOUNDARIES OF MONAD TENSORABILITY ON SET
}

\author{
NATHAN BOWLER $^{a}$, SERGEY GONCHAROV $^{b}$, PAUL BLAIN LEVY $^{c}$, AND LUTZ SCHRÖDER $^{d}$ \\ ${ }^{a}$ Department of Mathematics, Universität Hamburg \\ e-mail address: N.Bowler1729@gmail.com \\ ${ }^{b, d}$ Department of Computer Science, Friedrich-Alexander-Universität Erlangen-Nürnberg \\ e-mail address: \{Sergey.Goncharov, Lutz.Schroeder\}@fau.de \\ ${ }^{c}$ School of Computer Science, University of Birmingham \\ e-mail address: P.B.Levy@cs.bham.ac.uk
}

\begin{abstract}
We study a composition operation on monads, equivalently presented as large equational theories. Specifically, we discuss the existence of tensors, which are combinations of theories that impose mutual commutation of the operations from the component theories. As such, they extend the sum of two theories, which is just their unrestrained combination. Tensors of theories arise in several contexts; in particular, in the semantics of programming languages, the monad transformer for global state is given by a tensor. We present two main results: we show that the tensor of two monads need not in general exist by presenting two counterexamples, one of them involving finite powerset (i.e. the theory of join semilattices); this solves a somewhat long-standing open problem, and contrasts with recent results that had ruled out previously expected counterexamples. On the other hand, we show that tensors with bounded powerset monads do exist from countable powerset upwards.
\end{abstract}

\section{INTRODUCTION}

The concept of monad may be regarded as a category-theoretic abstraction of the notion of equational theory that is insensitive to the choice of syntax. More recently, monads have gained importance in the theory and practice of programming, where they are now commonly recognized as a standard formal abstraction for computational effects. In this context, the combination of theories, or monads, can be seen as modelling the combination of computational effects, a topic of interest e.g. in the modular semantics of programming languages.

One way to implement the combination of effects is via monad transformers [3, 19]. Essentially, a monad transformer is a function that sends monads to monads (additional

2012 ACM CCS: [Theory of computation]: Logic-Equational logic and rewriting; Semantics and reasoning-Program reasoning; Semantics and reasoning-Program semantics - Categorical semantics.

Key words and phrases: Monads, tensor products, side effects, non-determinism.

${ }^{c}$ Paul Blain Levy supported by EPSRC Advanced Research Fellowship EP/E056091. 
properties, such as functoriality, are not in general imposed). Monad transformers form the core of the treatment of side-effects in the functional programming language Haskell [21]. Besides the fact that due to their lack of structure they say only very little about the mathematical foundations of monad combination, monad transformers have been criticized for their asymmetry [12] which treats one set of effects as the monad transformer and the other set of effects as an argument of the latter. E.g. the approach via monad transformers hides the symmetry in the combination of exceptions and I/O: this combination can be obtained either by applying the I/O monad transformer to the exception monad or by applying the exception monad transformer to the $\mathrm{I} / \mathrm{O}$ monad, but this equivalence is not apparent from the corresponding monad transformer expressions. Moreover, monad transformers are ad hoc in character, and have been known only for a limited number of effects, a prominent negative example being nondeterminism.

It has turned out, however, that some of the most important monad transformers have an elegant abstract description using sum and tensor. Specifically, the monad transformers for exceptions and I/O constructs a sum, and the monad transformers for state, reader, and writer construct a tensor [12, 11. Whereas the sum of two monads is the simplest monad supporting both given effects without any interaction between them (and corresponds, in terms of theories, just to taking the disjoint union), the tensor (whose definition goes back to [7]) moreover requires commutation of these effects over each other, e.g. in case of tensoring statefulness with finite nondeterminism one has

$$
(\mathrm{x}:=\mathrm{a} ;(\mathrm{b}+\mathrm{c}))=((\mathrm{x}:=\mathrm{a} ; \mathrm{b})+(\mathrm{x}:=\mathrm{a} ; \mathrm{c}))
$$

and the like. We refer to the general form of this condition as the tensor law. When we view monads as representations of theories, the tensor law just states that the operations of one theory are homomorphic with respect to those of the other theory, i.e. it describes algebras (also known as "models") of the first theory in the category of algebras of the second.

As indicated above, an important example of the tensor product $\otimes$ is tensoring with global state, in which case the result is equivalent to application of the state monad transformer 23], e.g. $\mathcal{P} \otimes T_{S}=S \rightarrow \mathcal{P}\left(S \times{ }_{-}\right)$where $\mathcal{P}$ is the powerset monad and $T_{S}=S \rightarrow\left(S \times \times_{-}\right)$is the (global) state monad. One can look at this case from the opposite perspective and consider it as an application of a nondeterminism monad transformer $T \mapsto \mathcal{P} \otimes T$. This transformer yields the universal completely additive monad over $T$ [10], which therefore allows for a generalized Fischer-Ladner decomposition of control operators, i.e. roughly the translation

$$
\begin{aligned}
\text { if } b \text { then } p \text { else } q & :=b ? ; p+(\neg b) ? ; q \\
\text { while } b \text { do } p & :=(b ? ; p)^{\star} ;(\neg b) ?
\end{aligned}
$$

The catch in all this is that unless one requires both component monads to be ranked, i.e. generated by a set of algebraic operations, there is no guarantee that sum and tensor exist [12]. Intuitively, unranked monads arise when the number of values that can participate in a computation is unbounded. Unbounded non-determinism and continuations are prominent examples of unranked monads; in particular, it has long been unclear that the above-mentioned non-determinism monad transformer actually exists. It is comparatively easy to see that the sum of simple ranked monads with most unranked monads will typically fail to exist (see, e.g., [13]). The case of tensoring is more subtle. For the specific example of the (unranked) continuation monad, it has been shown in [11] that the tensor does exist if the partner monad is ranked. It has been conjectured in op. cit. (p. 30) that the tensor 
of unranked monads does not exist in general, and it has been implicitly indicated (op. cit., p. 33) that the tensor of continuations with a suitable unranked monad might serve as a counterexample, which seemed reasonable insofar as continuations generally constitute a good source of counterexamples (see, e.g., [24]).

However, two of us (Goncharov and Schröder) have recently proved that the tensor of two monads always exists if one of them is uniform, a natural criterion that ensures sufficiently pervasive applicability of the tensor law [9]. The class of uniform monads is surprisingly broad and includes not only countable and unbounded non-determinism (which implies that the above-mentioned non-determinism monad transformer does after all exist), but also continuations, thus discharging the latter as a suspect for a potential counterexample to existence of tensors. In summary, prior to the current work (respectively the conference version [8]), the question of universal existence of monad tensors was open, and no good candidates for possible counterexamples were known. It should be noted that the question as such dates back at least to [17], where it appears in the context of early developments of the categorical foundations of universal algebra.

Having said this, we do settle the question in the negative in the present work. Specifically, we present two countexamples to tensorability. By the above, at least one of the partner monads in a counterexample must be unranked, and in both our examples, the other partner is in fact ranked. One of these, originally presented in [8], shows that the tensor of a well-order monad with a simple free algebra monad (with two binary operations) fails to exist. In the other example, the ranked partner is finite powerset, while the unranked partner is less easy to grasp, being defined by a rather involved equational theory.

Moreover, we settle tensorability of non-empty $\kappa$-bounded powerset $\mathcal{P}_{\kappa}^{\star}$ in the positive in the remaining cases. Specifically, the uniformity method of [9] proves tensorability of $\mathcal{P}_{\kappa}^{\star}$ for all successor cardinals $\kappa$, and as mentioned above we show in the present work that tensorability fails for $\kappa=\omega$. We prove that $\mathcal{P}_{\kappa}^{\star}$ is tensorable for every uncountable $\kappa$; the proof method is dedicated to this case and does not currently seem to generalize to other monads, except that tensorability of full bounded powerset follows immediately in the applicable cases.

The paper is organised as follows. We give an introduction to monads and their algebras as well as their use in programming language semantics in Section 2. In Section 3, we discuss tensors and tensor algebras, a notion that goes back to [17, and summarize known results (and simple new ones) on existence of tensors. In Section 4 , we prove tensorability of uncountably bounded powerset. We then proceed to prove our negative results in Sections 5 and 6. In both counterexamples, tensor algebras appear as a key technical tool in that we show non-existence of the tensor by exhibiting a family of reachable tensor algebras of unbounded cardinality.

Note on Foundations. The category-theoretic concept of a monad on Set serves as an abstraction of the notion of equational theory. The constructions involved are well-known in the ranked case; we will discuss the situation for the unranked case in detail. To formalize the correspondence between theories and monads, we shall need to consider theories with large signatures.

We accordingly work in the von Neumann-Bernays-Gödel (NBG) theory of sets and classes, which is conservative over ZFC set theory [6]. In NBG, certain classes are sets, whilst others, such as the class of sets or the class of ordinals, are proper classes. All 
elements of classes are sets. We shall use "small" to indicate that something is a set, and "large" to indicate that it might be a proper class.

We shall also speak of "hyperlarge" categories such as the category Class of classes. Whereas a familiar large category has a class of objects, a hyperlarge category has a hyperclass of objects, and also a hyperclass of morphisms from one object to another. Informally, a hyperclass is a collection of classes. Formally, our statements involving hyperclasses are interpreted at the meta-level, in the same way that ZFC users would interpret statements involving classes: a hyperclass is the extension of a unary formula.

Note that, in NBG, care is needed to represent quotients and tuples of classes in such a way that they are classes.

- To quotient a class by an equivalence relation, we may either use the Global Axiom of Choice ("the class of sets is well-orderable") to represent each equivalence class by a chosen element, or employ "Scott's trick" 25] of representing each equivalence class by its set of elements of least rank.

- Following Morse [20] an ordered pair $(X, Y)$ of classes is represented as the class $X+Y$. More generally a large (i.e. class-indexed) tuple of classes $\left(X_{i} \mid i \in I\right)$ is represented as the class $\sum_{i \in I} X_{i}$. Consequently a hyperlarge sum or large product of hyperclasses is a hyperclass, just as a large sum or small product of classes is a class.

There are numerous other foundational options besides the one we have chosen, e.g. developing a theory of hyperclasses conservative over NBG; interpreting all our statements directly as properties of constructions on ZFC formulas; or using ZFC with one or even two Grothendieck universes, at the cost of losing conservativity over ZFC.

\section{Monads And Theories}

In programming language semantics, monads serve to encapsulate side-effects, a principle originally due to Moggi [18 that was subsequently introduced into the functional programming language Haskell as the principal means of dealing with impure features [26]. In a nutshell, the idea is to relocate the side effect from the function arrow into the result type of a function: a side-effecting function $X \rightarrow Y$ becomes a pure function $X \rightarrow T Y$, where $T Y$ is a type of side-effecting computations over $Y$; the base example is $T Y=S \rightarrow(S \times Y)$ for a fixed set $S$ of states, so that functions $X \rightarrow T Y$ are functions that may read and update a global state.

Formally, a monad on Set consists of a functor $T$ : Set $\rightarrow$ Set mapping sets $X$ (of values or, from the point of view of theories, variables) to sets $T X$ (of computations, or terms modulo equations) and two natural transformations $\eta:$ id $\rightarrow T$ and $\mu: T^{2} \rightarrow T$, the unit and the multiplication, respectively, subject to the equations $\mu \eta_{T}=\mu T \eta=\mathrm{id}$ and $\mu T \mu=\mu \mu_{T}$. We usually denote a monad by just its functor part $T$, with the other components understood implicitly. On Set, any monad has a unique strength, i.e. comes equipped with a unique natural transformation $X \times T Y \rightarrow T(X \times Y)$ satisfying a number of conditions [18. A monad morphism is a natural transformation between the underlying monad functors satisfying obvious conditions with respect to the unit, the Kleisli extension (equivalently, multiplication) see e.g. 2] for details. For monads on Set preservation of strength is automatic.

A monad $T$ on Set induces two categories, the smallest and the largest realization of $T$ as an adjunction, respectively: the Kleisli category Set $_{T}$ of $T$ has sets as objects and maps $X \rightarrow T Y$ as morphisms; the unit $\eta: X \rightarrow T X$ serves as the identity on $X$, and composition 
is given by Kleisli composition $(f, g) \mapsto \mu(T f) g$. On the other hand, the Eilenberg-Moore category Set $^{T}$ of $T$ consists of $T$-algebras, which are maps of the form $\alpha: T X \rightarrow X$ such that $\alpha \eta_{X}=\operatorname{id}_{X}$ and $\alpha \mu_{X}=\alpha T \alpha$, and their morphisms. Here, a morphism $f: \alpha \rightarrow \beta$ of $T$-algebras $\alpha: T X \rightarrow X$ and $\beta: T Y \rightarrow Y$ is a map $f: X \rightarrow Y$ such that $f \alpha=\beta T f$.

We now turn to theories. A large signature $\Sigma$ is a class $\Sigma$ of operations $f$ each with an associated small arity $\alpha(f)$. A large theory $\mathcal{T}=(\Sigma, \mathcal{E})$ consists of a large signature $\Sigma$ and a class $\mathcal{E}$ of equations between $\Sigma$-terms. (Formally, an equation is a tuple $\left(X, t, t^{\prime}\right)$ where $X$ is a set and $t$ and $t^{\prime}$ are $\Sigma$-terms over $X$.) An algebra for such a theory is a class equipped with a $\Sigma$-structure satisfying all of the equations in $\mathcal{E}$. The algebra is termed small if its carrier (not the algebra as a whole) is a set. For each set $X$ we build the free algebra $F_{\mathcal{T}}(X)$ on $X$ as the class of $\Sigma$-terms over $X$ taken modulo the equations in $\mathcal{T}$ : this may fail to be small.

More generally, for a category $\mathcal{C}$ with small products, a $\mathcal{T}$-algebra in $\mathcal{C}$ consists of an object $A$ of $\mathcal{C}$ together with a map $A^{\alpha(f)} \stackrel{f^{A}}{\longrightarrow} A$ for each $f \in \Sigma$ such that, for each equation $\left(X, t, t^{\prime}\right) \in \mathcal{E}$, the maps $A^{X} \rightarrow A$ corresponding to the two terms being equated are equal. An algebra homomorphism from $A$ to $B$ consists of a map $A \stackrel{k}{\rightarrow} B$ in $\mathcal{C}$ commuting with all the operations in the sense that for any $f \in \Sigma$ we have $f^{A} \cdot k^{\alpha(f)}=k \cdot f$. The category of $\mathcal{T}$-algebras in $\mathcal{C}$ is denoted $\mathcal{T}$ - $\mathbf{A l g}[\mathcal{C}]$. There is an evident forgetful functor from $\mathcal{T}$ - $\mathbf{A l g}[\mathcal{C}]$ to $\mathcal{C}$, which creates small products.

Returning to the category of sets, $\mathcal{T}$-Alg $[\mathbf{S e t}]$ is the category of small $\mathcal{T}$-algebras, and there is an evident forgetful functor $U_{\mathcal{T}}$ from it to Set. There are two ways in which $U_{\mathcal{T}}$ can have a left adjoint (in which case it is even monadic):

Definition 2.1. A large theory $\mathcal{T}$ has small free algebras if $F_{\mathcal{T}}(X)$ is small for every set $X$, and free small algebras if $U_{\mathcal{T}}$ has a left adjoint.

It is, then, clear that

(1) every large theory $\mathcal{T}$ with small free algebras has free small algebras, where the left adjoint of $U_{\mathcal{T}}$ maps $X$ to $F_{\mathcal{T}}(X)$;

(2) every large theory $\mathcal{T}$ with free small algebras gives rise to, or presents, a monad in the standard way, i.e. by composing $U_{\mathcal{T}}$ with its left adjoint,

i.e. we have

$$
\text { Large theories }
$$

where the arrow $\rightarrow$ is intended to denote a map. We make three observations about (2.1). Firstly the inclusion is strict.

Theorem 2.2. There exists a large theory that has free small algebras but not small free algebras.

Proof. Let $\mathcal{T}$ be the following theory. For the signature we take a constant $k_{\alpha}$ for each ordinal $\alpha$ and a function $f$ of arity 3. For the equations we take $f\left(k_{\alpha}, k_{\alpha}, x\right)=k_{0}$ for any $\alpha$, and $f\left(k_{\alpha}, k_{\beta}, x\right)=x$ for any distinct $\alpha, \beta$. Then the elements of $F_{\mathcal{T}}(\varnothing)$ are in bijection with the set of constants $k_{\alpha}$, with the action of $f$ completely determined by the equations in $\mathcal{T}$, so $F_{\mathcal{T}}(\varnothing)$ fails to be small, i.e. $\mathcal{T}$ does not have small free algebras. On the other hand, in any small $\mathcal{T}$-algebra $A$, the interpretations of $k_{\alpha}$ and $k_{\beta}$ must be equal for some pair of distinct ordinals $\alpha, \beta$. Then for any $x$ in $A$ we have $x=f\left(k_{\alpha}, k_{\beta}, x\right)=f\left(k_{\alpha}, k_{\alpha}, x\right)=k_{0}$, so that $A$ has only one element. Hence $U_{\mathcal{T}}$ has a left adjoint, i.e. $\mathcal{T}$ has free small algebras. 
Suppose $\mathcal{T}$ is a theory with free small algebras that presents the monad $T$. We call $\mathcal{T}$ a genuinely presenting theory of $T$ if $\mathcal{T}$ has small free algebras, and otherwise a spuriously presenting theory. Thus the theory in the preceding proof spuriously presents the monad $X \mapsto 1$. We next see that every monad has a genuinely presenting theory.

Definition 2.3. Let $\left(T, \eta,-^{*}\right)$ be a monad on Set expressed as a Kleisli triple. We define the large theory $\Psi T$ as follows. For the signature, we take for every set $X$ and $m \in T X$ an operation $h_{X, m}$ of arity $X$. For the equations, we take

- for every set $X$ and $a \in X$, an equation

$$
h_{X, \eta_{X}(a)}\left(p_{x} \mid x \in X\right)=p_{a} .
$$

- for all sets $X, Y$ and $m \in T X, f: X \rightarrow T Y$, an equation

$$
h_{X, m}\left(h_{Y, f(x)}\left(p_{y} \mid y \in Y\right) \mid x \in X\right)=h_{Y, f^{*} m}\left(p_{y} \mid y \in Y\right) .
$$

Theorem 2.4. Any monad $T$ on Set is genuinely presented by the large theory $\Psi T$. Thus we have

$$
\text { Set }^{T} \cong_{\text {Set }} \quad \Psi T \text {-Alg }[\text { Set }]
$$

where $\cong_{\text {Set }}$ indicates an isomorphism commuting with the forgetful functors to Set.

We exploit the theory $\Psi T$ to define algebras in other categories.

Definition 2.5. Let $T$ be a monad on Set. For any category with small products $\mathcal{C}$, we define the $T$-algebras in $\mathcal{C}$ via

$$
T-\mathbf{A l g}[\mathcal{C}] \stackrel{\text { def }}{=} \Psi T-\mathbf{A l g}[\mathcal{C}]
$$

Remark 2.6. $T$-Alg $[\mathcal{C}]$ is equivalent to the category of functors from $\mathbf{S e t}_{T}^{o p}$ to $\mathcal{C}$ preserving small products. The latter was used for the same purpose in [5, 11].

We close the circle by seeing that the composite (2.1) does not affect the category of algebras.

Theorem 2.7. Let $T$ be a monad on Set genuinely presented by $\mathcal{T}$. For any category $\mathcal{C}$ with small products, we have

$$
\mathcal{T}-\operatorname{Alg}[\mathcal{C}] \quad \cong_{\mathcal{C}} \quad T-\operatorname{Alg}[\mathcal{C}]
$$

Theorem 2.7 tells us that the category of algebras depends only on the monad, not on the choice of genuinely presenting theory. With these conversions in place, we will switch back and forth freely between monads and large theories as convenient. (Monads are also formally equivalent to large Lawvere theories [15], which were used in the theory of generic side-effects in [11].)

The following easy results will be useful.

\section{Proposition 2.8.}

(1) Let $T_{0}$ be a monad on Set genuinely presented by $\mathcal{T}=(\Sigma, \mathcal{E})$, and $\alpha: T_{0} \rightarrow T_{1}$ a componentwise surjective monad morphism. Then $T_{1}$ is genuinely presented by the theory with signature $\Sigma$ and equation class

$$
\mathcal{E} \cup\left\{\left(X, t, t^{\prime}\right) \mid \alpha_{X}[t]_{\mathcal{E}}=\alpha_{X}\left[t^{\prime}\right]_{\mathcal{E}}\right\}
$$

(2) Let $T$ be a monad on Set genuinely presented by $\mathcal{T}=(\Sigma, \mathcal{E})$. For any set $E$, the monad $T(-+E)$ is genuinely presented by the theory with signature $\Sigma$ extended by a family of constants $\left(c_{e} \mid e \in E\right)$, and equation class $\mathcal{E}$. 
The notion of rank for a monad refers to the arity of the involved algebraic operations. Formally, a monad is $\kappa$-ranked for a regular cardinal $\kappa$ if the underlying functor preserves $\kappa$-filtered colimits. A monad is ranked if it is $\kappa$-ranked for some $\kappa$. The $\kappa$-ranked monads on Set are precisely those that are induced by a small theory in which the arity of each operation is less than $\kappa$, so that the ranked monads are precisely those induced by small theories. Computationally relevant unranked monads include the continuation monad and the unbounded powerset and are presented further below. It has been shown that the algebraic view of ranked monads gives rise to computationally natural operations; e.g. the state monad (with state set $S=V^{L}$ for sets $V$ of values and $L$ of locations) can be algebraically presented in terms of operations lookup and update 22.

We give some standard examples of computational monads, mostly from [18]:

Example 2.9 (Computational Monads).

(1) Global state: as stated initially, $T X=S \rightarrow(S \times X)$ is a monad (for this and other standard examples, we omit the description of the remaining data), the well-known state monad.

(2) Nondeterminism: the generic (unranked) monad for nondeterminism is the one presented by the covariant powerset functor $\mathcal{P}$. Variants arise on the one hand by restricting to nonempty subsets, thus ruling out non-termination, and on the other hand by bounding the cardinality of subsets. We denote nonemptyness by a superscript $\star$, and cardinality bounds by subscripts. E.g., the monad $\mathcal{P}_{\omega_{1}}^{\star}$ describes countable non-blocking nondeterminism. Yet another variant arises by replacing sets with countable multisets, i.e. maps $X \rightarrow(\mathbb{N} \cup\{\infty\})$, thus modelling weighted nondeterminism [4]. Let us denote by $T_{\text {mult }}$ the corresponding countable multiset monad.

(3) Continuations: The continuation monad maps a set $X$ to the set $(X \rightarrow R) \rightarrow R$, for a fixed set $R$ of results. We denote the corresponding unranked monad as $T_{\text {cont }}^{R}$.

(4) Input/Output: For a given set $I$ of input symbols, the monad $T_{I}$ for input is generated by a single $I$-ary operation; this monad is induced by an absolutely free theory, i.e. one without equations. Similarly, given a set $O$ of output symbols, the monad $T_{O}$ for output is induced by a family of unary operations indexed over $O$.

A convenient way of denoting generic computations is the so-called computational metalanguage [18], which has found its way into functional programming in the shape of Haskell's do-notation. We briefly outline the version of the metalanguage we use below; this version is deliberately simplistic, as it serves only to elucidate the definition of tensors.

The metalanguage denotes morphisms in the underlying category of a given monad, using the monadic structure; since we are working over Set, the metalanguage just denotes maps in our setting. We let a signature $\Sigma$ consist of a set $\mathcal{B}$ of base types, to be interpreted as sets, and a collection of typed function symbols $f: A_{1} \rightarrow A_{2}$ to be interpreted as functions, where $A_{1}, A_{2}$ are types. Here, we assume that the set $\mathcal{T}$ of types is generated from the base types by the grammar

$$
\mathcal{T} \ni A_{1}, A_{2}::=1|B| A_{1} \times A_{2} \mid T A_{1} \quad(B \in \mathcal{B})
$$

where $\times$ is interpreted as set theoretic product, 1 is a singleton set, and $T$ is application of the given monad. We then have standard formation rules for terms-in-context $\Gamma \triangleright t: A$, read 'term $t$ has type $A$ in context $\Gamma$ ', where a context is a list $\Gamma=\left(x_{1}: A_{1}, \ldots, x_{n}: A_{n}\right)$ of typed variables (later, contexts will mostly be omitted): 


$$
\begin{aligned}
& \frac{x: A \in \Gamma}{\Gamma \triangleright x: A} \quad \frac{f: A \rightarrow B \in \Sigma \quad \Gamma \triangleright t: A}{\Gamma \triangleright f(t): B} \quad \overline{\Gamma \triangleright \star: 1} \\
& \frac{\Gamma \triangleright s: A \quad \Gamma \triangleright t: B}{\Gamma \triangleright\langle s, t\rangle: A \times B} \quad \frac{\Gamma \triangleright t: A \times B}{\Gamma \triangleright \mathrm{fst} t: A} \quad \frac{\Gamma \triangleright t: A \times B}{\Gamma \triangleright \mathrm{snd} t: B} \\
& \frac{\Gamma \triangleright t: A}{\Gamma \triangleright \operatorname{ret} t: T A} \quad \frac{\Gamma \triangleright p: T A \quad \Gamma, x: A \triangleright q: T B}{\Gamma \triangleright \operatorname{do} x \leftarrow p ; q: T B}
\end{aligned}
$$

Only the operations in the last line are specific to monads; they are called return and binding, respectively. For binding, we use Haskell's do-notation. Return is interpreted by the unit $\eta$ of the monad, and can be thought of as returning a value. A binding do $x \leftarrow p$; $q$ executes $p$, binds its result to $x$, and then executes $q$, which may use $x$. Binding is right associative, i.e. do $x \leftarrow p ; y \leftarrow q ; r=$ do $x \leftarrow p$; (do $y \leftarrow q ; r$ ). It is interpreted using Kleisli composition and strength, where the latter serves to propagate the context $\Gamma[18$. In consequence, one has the monad laws

$$
\begin{gathered}
\text { do } x \leftarrow p \text {; ret } x=p \quad \text { do } x \leftarrow \operatorname{ret} a ; p=p[a / x] \\
\text { do } x \leftarrow(\text { do } y \leftarrow p ; q) ; r=\text { do } y \leftarrow p ; x \leftarrow q ; r .
\end{gathered}
$$

Terms of a type $T A$ are called programs. We say that two programs $p: T A$ and $q: T B$ commute if they satisfy the equation

$$
\text { do } x \leftarrow p ; y \leftarrow q ; \operatorname{ret}\langle x, y\rangle=\text { do } y \leftarrow q ; x \leftarrow p ; \operatorname{ret}\langle x, y\rangle: T(A \times B) \text {. }
$$

Remark 2.10. The notion of commutation of programs relates as expected to the standard notion of commutative monad: a monad is commutative iff all its programs commute.

\section{Tensors and Tensor Algebras}

We begin by defining tensor of monads in terms of a universal property, just as the coproduct is defined to be an initial cocone.

Definition 3.1. Let $T$ and $S$ be monads on Set. A cocone from $T$ and $S$-that is to say, a monad $R$ together with morphisms $\tau: T \rightarrow R$ and $\sigma: S \rightarrow R$-is a commuting cocone when every two programs of the form $\tau_{X}(p)$ and $\sigma_{Y}(q)$ commute (see Section 2). A tensor product $T \otimes S$ is an initial commuting cocone, i.e. a commuting cocone from which there is a unique cocone morphism to any commuting cocone.

Just as the coproduct of monads corresponds to the disjoint union of theories [1, 12, 14, 16, so the tensor can be described in terms of theories.

Definition 3.2. Let $\mathcal{T}$ and $\mathcal{S}$ be large theories with signatures $\Sigma_{1}$ and $\Sigma_{2}$ respectively.

(1) Their disjoint union has as its signature the disjoint union of $\Sigma_{1}$ and $\Sigma_{2}$, and its equations consist of those of $\mathcal{T}$ together with $\mathcal{S}$. 
(2) The tensor product $\mathcal{T} \otimes \mathcal{S}$ of the theories is on the same signature as the disjoint union, and has as its equations all those of the disjoint union together with the commutativity equation

$$
f\left(g\left(x_{i j} \mid j \in \alpha(g)\right) \mid i \in \alpha(f)\right)=g\left(f\left(x_{i j} \mid i \in \alpha(f)\right) \mid j \in \alpha(g)\right)
$$

saying that $f$ and $g$ commute for any $f \in \Sigma_{1}$ and $g \in \Sigma_{2}$.

Remark 3.3. A theory $\mathcal{T}$ with signature $\Sigma$ is said to be commutative if all its operations are algebra homomorphisms, that is, if Equation (3.1) holds for all $f, g \in \Sigma$. Clearly, if $\mathcal{T}$ has small free algebras, then $\mathcal{T}$ is commutative iff the monad it presents is commutative.

Tensor algebras can also be viewed as algebras in categories of algebras.

Proposition 3.4. For large theories $\mathcal{T}$ and $\mathcal{S}$ and category $\mathcal{C}$ with small products, we have

$$
(\mathcal{T} \otimes \mathcal{S})-\operatorname{Alg}[\mathcal{C}] \quad \cong_{\mathcal{C}} \quad \mathcal{T}-\operatorname{Alg}[\mathcal{S}-\mathbf{A l g}[\mathcal{C}]]
$$

As in Section 2 we adapt tensor algebras from theories to monads.

Definition 3.5. Let $T$ and $S$ be monads on Set.

(1) [17] A small $(T, S)$-tensor algebra is a triple $(X, \alpha, \beta)$ where $X$ is a set and $\alpha$ and $\beta$ are respectively Eilenberg-Moore $T$ - and $S$-algebra structures on $X$, such that for all sets $Y, Z$ and all $p \in S Y, q \in T Z, f: Y \times Z \rightarrow X$, the following equation, called the tensor law, holds

$$
\beta\left(T\left(\lambda z . \alpha\left(S f_{-, z} p\right)\right) q\right)=\alpha\left(S\left(\lambda y \cdot \beta\left(T f_{y,-} q\right)\right) p\right)
$$

where $f_{-, z}(y)=f_{y_{-}}(z)=f(y, z)$ for $(y, z) \in Y \times Z$. Morphisms of $(T, S)$-tensor algebras are maps between the respective carriers which are homomorphic for both $T$ and $S$. The (large, locally small) category of $(T, S)$-tensor algebras is denoted $(T, S)$-TAlg.

(2) For any category with small products $\mathcal{C}$, we define

$$
(T, S)-\mathbf{T A l g}[\mathcal{C}] \stackrel{\text { def }}{=}(\Psi T \otimes \Psi S)-\mathbf{A l g}[\mathcal{C}]
$$

We then have the following counterpart of Theorems 2.4 and 2.7 .

Theorem 3.6. Let $T$ and $S$ be monads on Set.

(1) The two parts of Def. 3.5 agree: we have

$$
(T, S) \text {-TAlg } \cong_{\text {Set }}(T, S) \text {-TAlg }[\text { Set }]
$$

(2) Let $\mathcal{T}$ and $\mathcal{S}$ be genuinely presenting theories for $T$ and $S$ respectively. For any category $\mathcal{C}$ with small products, we have

$$
(\mathcal{T} \otimes \mathcal{S})-\mathbf{A l g}[\mathcal{C}] \quad \cong_{\mathcal{C}} \quad(T, S)-\mathbf{T A l g}[\mathcal{C}]
$$

Once again part (2) tells us that the category of tensor algebras depends only on the two monads, not on the choice of genuinely presenting theories.

Next we relate tensor algebras to the universal property of Def. 3.1. The following result appears (modulo translation from the language of large Lawvere theories into the language of monads) in [11].

Theorem 3.7. The tensor product of monads $T, S$ on Set exists if and only if the forgetful functor from $(T, S)$-TAlg to Set is monadic, equivalently has a left adjoint. In this case, the monad induced by the adjunction is the tensor $T \otimes S$. 
Remark and Definition 3.8. There are essentially three alternatives regarding the existence of a tensor of monads $T, S$ genuinely presented by large theories $\mathcal{T}, \mathcal{S}$.

(1) The tensor theory $\mathcal{T} \otimes \mathcal{S}$ may fail to have free small algebras, in which case $T \otimes S$ does not exist.

(2) $\mathcal{T} \otimes \mathcal{S}$ may have free small algebras but not small free algebras, in which case the tensor $T \otimes S$ does exist but does not have the expected form; we call such monad tensors spurious.

(3) $\mathcal{T} \otimes \mathcal{S}$ may have small free algebras, in which case $T \otimes S$ exists and has the expected form, i.e. maps a set $X$ to the underlying set of $F_{\mathcal{T} \otimes \mathcal{S}}(X)$; we call such monad tensors genuine.

By Theorem 3.6(2) with $\mathcal{C}=$ Class, this three-way classification must depend only on the monads $T$ and $S$ and not on the choice of genuinely presenting theories. We shall say that a monad $T$ on Set is tensorable when $T \otimes S$ exists for every monad $S$ on Set. If these tensors are all genuine, $T$ is genuinely tensorable.

It is currently an open question whether spurious monad tensors exist. Our results established below are always the stronger of the two possible variants: where we show existence of tensors, we actually show also that the tensor is genuine, and where we show non-existence, we prove that not even a spurious tensor exists.

The following is a straightforward consequence of Prop. 2.8 .

Proposition 3.9. (1) Let $T_{0}, T_{1}, T_{2}$ be monads on Set, and $\alpha: T_{0} \rightarrow T_{1}$ a componentwise surjective monad morphism. If $T_{0} \otimes S$ exists then so does $T_{1} \otimes S$ and the induced morphism $T_{0} \otimes S \rightarrow T_{1} \otimes S$ is componentwise surjective. Moreover if $T_{0} \otimes S$ is genuine then so is $T_{1} \otimes S$.

(2) Let $T$ and $S$ be monads on Set, and $E$ a set. If $T \otimes S$ exists then so does $T(-+E) \otimes S$ and the induced morphism $(T \otimes S)(-+E) \rightarrow T(-+E) \otimes S$ is componentwise surjective. Moreover if $T \otimes S$ is genuine then so is $T(-+E) \otimes S$.

Our negative results will be based on the following simple result.

Definition 3.10. As usual, we say that an algebra is generated by a subset $X$ if it does not have a proper subalgebra containing $X$. We say that an algebra is $\alpha$-reachable for a cardinal $\alpha$ if it has a generating set $X$ of cardinality $|X| \leq \alpha$.

Corollary 3.11. The (possibly spurious) tensor $T \otimes S$ of monads $T, S$ on Set exists if and only if for every cardinal $\alpha$, the cardinality of $\alpha$-reachable small $(T, S)$-tensor algebras is bounded.

Proof. 'Only if': If the tensor exists, then there is a free small $(T, S)$-tensor algebra over $\alpha$, and every $\alpha$-reachable small $(T, S)$-tensor algebra is a quotient of it.

'If': To show the forgetful functor from $(T, S)$-tensor algebras to Set has a left adjoint under the given assumption, we apply Freyd's general adjoint functor theorem, since every set carries only set-many small $(T, S)$-tensor algebras.

Prior to the current results, the state of research regarding counterexamples to tensorability was as follows. It is well-known that the tensor of two ranked monads on Set does exist [11, so that any counterexample needs to involve at least one unranked monad. One unranked monad that is known to show hard-to-control behaviour in many respects is the continuation monad. It has been shown in [11] that the tensor of any ranked monad with the continuation 
monad exists, and at the same time it has been conjectured that the continuation monad fails to be tensorable (i.e. that there exists an unranked monad whose tensor with the continuation monad fails to exist). However, it has subsequently been shown that all socalled uniform monads are tensorable [9], and the given proof in fact implies that the tensors in question are genuine. The class of uniform monads is quite broad and in particular includes both the powerset monad and the continuation monad, so that these monads are ruled out as counterexamples to tensorability.

\section{Tensoring With Bounded Powerset Monads}

As discussed in the last section, it has been shown using the uniformity method that the unbounded and countable powerset monads as well as their restrictions to non-empty subsets are tensorable [9], and we will show in Section 5 that the finite powerset monad fails to be tensorable. Right now, we will show that all non-empty uncountably bounded powerset monads, i.e. submonads of the powerset monad of the form $\mathcal{P}_{\kappa}^{\star}$, where $\mathcal{P}_{\kappa}^{\star}(X)$ denotes the set of non-empty subsets of $X$ of cardinality less than $\kappa$ for an uncountable regular cardinal $\kappa$, are genuinely tensorable. (Regularity of $\kappa$ is equivalent to $\mathcal{P}_{\kappa}^{\star}$ actually being a monad. Requiring $\kappa$ to be uncountable ensures that $\mathcal{P}_{\kappa}^{\star}$-algebras have countable joins.) Interestingly, the proof does not seem to relate to any generalization of uniformity. From genuine tensorability of $\mathcal{P}_{\kappa}^{\star}$, genuine tensorability of the full bounded powerset monad $\mathcal{P}_{\kappa}$ (which maps a set $X$ to the set of all subsets of $X$ of cardinality less than $\kappa$ ) is immediate by Proposition 3.9 (alternatively, the proof below can be adapted to full bounded powerset, and in fact becomes simpler in the process).

Let $T$ be a monad genuinely presented by $\mathcal{T}$, and let $X$ be a set. Let $A$ be the free large $\left(\mathcal{P}_{\kappa}^{\star}, T\right)$-tensor algebra on $X$; we need to show that $A$ is small. Let $\hat{X}$ be the image of $X$ in $A$. For any subset $Y$ of $A$, let $T^{\text {" }} Y$ denote the (necessarily small) sub- $T$-algebra of $A$ generated by $Y$, and similarly for $\mathcal{P}_{\kappa}^{\star}$. In general a $\mathcal{P}_{\kappa}^{\star}$-algebra is a semilattice in which every nonempty subset of size $<\kappa$ has a supremum; we denote by $\leq$ the ordering on $A$ induced by the $\mathcal{P}_{\kappa}^{\star}$-structure.

Lemma 4.1. For any $x \in A$ there is $t \in T$ " $\hat{X}$ such that $x \geq t$.

Proof. By induction on the complexity of terms. For any $x \in \hat{X}$, we have $x \geq x \in T$ " $\hat{X}$.

Next, let $x$ have the form $\bigvee_{i \in I} x_{i}$, where $I$ is a nonempty set of cardinality smaller than $\kappa$, and pick $i \in I$. By the induction hypothesis, we have some $t \in T$ " $\hat{X}$ with $t \leq x_{i} \leq x$.

Finally, let $x$ have the form $f\left(x_{j} \mid j \in \alpha(f)\right)$ for some operation $f$ of $\mathcal{T}$. By the induction hypothesis we can pick $\left(t_{j} \in T^{\text {" }} \hat{X} \mid j \in \alpha(f)\right)$ with $t_{j} \leq x_{j}$ for each $j \in \alpha(f)$. Then $f\left(x_{j} \mid j \in \alpha(f)\right)=f\left(x_{j} \vee t_{j} \mid j \in \alpha(f)\right)=f\left(x_{j} \mid j \in \alpha(f)\right) \vee f\left(t_{j} \mid j \in \alpha(f)\right)$, so that $x \geq f\left(t_{j} \mid j \in \alpha(f)\right) \in T^{\text {" }} T$ " $\hat{X}=T$ " $\hat{X}$.

Now define a sequence of subsets $\left(X_{n} \mid n \in \mathbb{N}\right)$ of $A$ by $X_{0}=\hat{X}$ and $X_{n+1}=\mathcal{P}_{\kappa}^{\star}$ " $T$ " $X_{n}$, and let $X_{\omega}=\bigcup_{n \in \mathbb{N}} X_{n}$.

Lemma 4.2. Any $x \in \mathcal{P}_{\kappa}^{\star}$ " $X_{\omega}$ can be written in the form $\bigvee_{n \in \mathbb{N}>0} x_{n}$, with each $x_{n} \in X_{n}$

Proof. We can write $x$ in the form $\bigvee_{i \in I} x_{i}$ with $I$ nonempty and of cardinality $<\kappa$ and $x_{i} \in X_{\omega}$ for all $i$. For $n \in \mathbb{N}_{>0}$, let $I_{n}=\left\{i \in I \mid x_{i} \in X_{n}\right\}$, so that $\bigcup_{n \in \mathbb{N}>0} I_{n}=I$. Pick (by Lemma 4.1) $t \in T$ " $\hat{X}$ such that $t \leq x$, and $n_{0} \in \mathbb{N}_{>0}$ minimal such that $I_{n}$ is nonempty. 
Taking $x_{n}=t \in T^{\text {" }} \hat{X} \subseteq X_{n}$ for $0<n<n_{0}$ and $x_{n}=\bigvee_{i \in I_{n}} x_{i}$ for $n \geq n_{0}$, we obtain $x=\bigvee_{n \in \mathbb{N}>0} x_{n}$, as required.

Lemma 4.3. $T$ " $\mathcal{P}_{\kappa}^{\star}$ " $X_{\omega} \subseteq \mathcal{P}_{\kappa}^{\star}$ " $X_{\omega}$

Proof. By Lemma 4.2, any $x \in T$ " $\mathcal{P}_{\kappa}^{\star}$ " $X_{\omega}$ can be expressed as $f\left(\bigvee_{n \in \mathbb{N}_{>0}} x_{i, n} \mid i \in \alpha(f)\right)$ for some operation $f$ of $T$ and $x_{i, n} \in X_{n}$. Commutation of the $\mathcal{P}_{\kappa}^{\star}$-structure with $f$ implies $x=\bigvee_{n \in \mathbb{N}_{>0}} f\left(x_{i, n} \mid i \in \alpha(f)\right) \in \mathcal{P}_{\kappa}^{\star}$ " $X_{\omega}$ since for each $n \in \mathbb{N}_{>0}$ we have $f\left(x_{i, n} \mid i \in \alpha(f)\right) \in$ $T$ " $X_{n} \subseteq X_{n+1} \subseteq X_{\omega}$.

It follows that $A=T$ " $\mathcal{P}_{\kappa}^{\star}$ " $X_{\omega}$, and in particular $A$ is small. Since $X$ was arbitrary, this implies that the monad tensor $\mathcal{P}_{\kappa}^{\star} \otimes T$ exists, and since $T$ was arbitrary, we obtain (using Proposition 3.9 again)

Theorem 4.4. For every regular cardinal $\kappa>\omega, \mathcal{P}_{\kappa}^{\star}$ and $\mathcal{P}_{\kappa}$ are genuinely tensorable.

\section{Finite Powerset Fails to Be Tensorable}

We now turn to our negative results, i.e. examples of two monads whose tensor fails to exist. Necessarily, one of these must be unranked. In this section and the next, we present two examples of this kind. The first, to be discussed presently, involves the finite powerset monad, which is computationally significant as a monadic model of finite non-determinism; the unranked partner in the example is a somewhat involved theory that we explain in detail below. The second example involves to comparatively simple monads, a type of well-order monad and a free monad; this was actually the first example to be found [8].

In both cases, we construct the unranked partner monad via a large theory. First, we introduce the theory and show that has small free algebras, and hence induces a monad. Then we show that the tensor product with the (small) theory of the ranked partner has arbitrarily large tensor algebras all generated by some particular set. The fact that this tensor product does not induce a monad is then immediate from Corollary 3.11 .

We shall think of $\mathcal{P}_{\omega}$ as the 'free bounded semilattice' monad, that is as the monad corresponding to the theory of bounded semilattices.

We now define the unranked partner monad $\mathcal{M}$ as induced by a large theory $\mathcal{T}_{\mathcal{M}}$. The signature $\Sigma$ of $\mathcal{T}_{\mathcal{M}}$ is defined as follows. For any ordinal $\alpha$, we define $\alpha^{+}$to be the least regular ordinal greater than $\alpha$. Given a ordinal $\delta$, let $B_{\delta}$ be the set of all triples $(\alpha, \beta, i)$ of ordinals such that $\alpha<\delta, \beta<\alpha^{+}, i<\omega$. Let $\Sigma_{\kappa}$ be the signature consisting of a constant symbol $c$ and operation symbols $f_{\alpha, \beta, i}$ of arity $B_{\alpha}$ for each $(\alpha, \beta, i) \in B_{\kappa+1}, \alpha>0$ and let $\Sigma$ be the (large) union of all the $\Sigma_{\kappa}$.

The theory $\mathcal{T}_{\mathcal{M}}$ consists of equations

$$
f_{\alpha, \beta, i}\left(t_{\alpha^{\prime}, \beta^{\prime}, i^{\prime}} \mid\left(\alpha^{\prime}, \beta^{\prime}, i^{\prime}\right) \in B_{\alpha}\right)=c
$$

whenever the $t_{\alpha^{\prime}, \beta^{\prime}, i^{\prime}}$ are terms in variables drawn from some set $X$ of cardinality strictly smaller than that of $\alpha$.

We may understand algebras for this theory as follows. Given a $\Sigma$-algebra $A$, a set $X$, a map $x: X \rightarrow A$, and $\Sigma^{\prime} \subseteq \Sigma$, let us denote by $\langle x\rangle_{\Sigma^{\prime}}$ the $\Sigma^{\prime}$-subalgebra of $A$ generated by the image of $x$. Then a $\mathcal{T}_{\mathcal{M}}$-algebra is just a $\Sigma$-algebra that satisfies the following property: Given a cardinal $\kappa$, a set $X$ of cardinality $\kappa$ and a map $x: X \rightarrow A$, the equation

$$
f_{\alpha, \beta, i}\left(a_{\alpha^{\prime}, \beta^{\prime}, i^{\prime}} \mid\left(\alpha^{\prime}, \beta^{\prime}, i^{\prime}\right) \in B_{\alpha}\right)=c
$$


holds over $\langle x\rangle_{\Sigma_{\kappa}}$ whenever $\alpha>\kappa$. Note that this implies

$$
\langle x\rangle_{\Sigma}=\langle x\rangle_{\Sigma_{\kappa}} .
$$

Lemma 5.1. The theory $\mathcal{T}_{\mathcal{M}}$ has small free algebras.

Proof. The free algebra over a set $X$ satisfies (5.3) for $\kappa=|X|$.

We denote the monad induced by $\mathcal{T}_{\mathcal{M}}$ by the above lemma by $\mathcal{M}$. We proceed to construct a sequence of reachable $\mathcal{T}_{\mathcal{M}}$-algebras of unbounded cardinality. For any cardinal $\delta$, put $M_{\delta}=\mathcal{P}_{\omega}\left(B_{\delta}\right)$. For the definition of the $\Sigma$-algebra structure, we need a few technical preliminaries.

Definition 5.2. Let $X$ be a set, and let $\left(x_{i} \mid i \in \omega\right)$ be a sequence of elements of $X$. We say that a sequence $\left(a_{i} \mid i \in \omega\right)$ of finite subsets of $X$ subsumes $\left(x_{i} \mid i \in \omega\right)$ if and only if there is an infinite subset $M$ of $\omega$ such that for any $i, j \in M$ with $i<j$ we have $x_{i} \in a_{j}$.

It will be helpful to make use of an unusual sort of quantifier: we use $\exists_{c f} \beta<\alpha$. $\phi(\beta)$ to mean 'the set of $\beta<\alpha$ such that $\phi(\beta)$ holds is cofinal in $\alpha$ 1 . We put a $\Sigma$-algebra structure on $M_{\delta}$ by taking $f_{\alpha, \beta, i}\left(a_{\alpha^{\prime}, \beta^{\prime}, i^{\prime}} \mid\left(\alpha^{\prime}, \beta^{\prime}, i^{\prime}\right) \in B_{\alpha}\right)$ to be

$$
\begin{aligned}
&\{(\alpha, \beta, i)\} \quad \text { if } \alpha<\delta \text { and } \\
& \exists_{c f} \alpha^{\prime}<\alpha . \exists_{c f} \beta^{\prime}<\alpha^{\prime+} .\left(a_{\alpha^{\prime}, \beta^{\prime}, i^{\prime}} \mid i^{\prime} \in \omega\right) \text { subsumes }\left(\left(\alpha^{\prime}, \beta^{\prime}, i^{\prime}\right) \mid i^{\prime} \in \omega\right) \\
& \varnothing \quad \text { otherwise }
\end{aligned}
$$

and interpreting $c$ as the empty set. We proceed to show that this does indeed define an $\mathcal{T}_{\mathcal{M}}$-algebra.

Lemma 5.3. If $\left(a_{i} \mid i \in \omega\right)$ subsumes a sequence $\left(x_{i} \mid i \in \omega\right)$ of distinct elements of $X$ then some $a_{i}$ contains at least two elements of that sequence.

Lemma 5.4. For every $\delta, M_{\delta}$ is a $\mathcal{T}_{\mathcal{M}}$-algebra.

Proof. Suppose for a contradiction that $f_{\alpha, \beta, i}\left(a_{\alpha^{\prime}, \beta^{\prime}, i^{\prime}} \mid\left(\alpha^{\prime}, \beta^{\prime}, i^{\prime}\right) \in B_{\alpha}\right) \neq c=\varnothing$ with $a_{\alpha^{\prime}, \beta^{\prime}, i^{\prime}} \in\langle x\rangle_{\Sigma^{\kappa}}$ for some $x: X \rightarrow M_{\delta}$ where $|X|=\kappa$ and $\alpha>\kappa$. Then $(\alpha<\delta$ and $)$

$$
\exists_{c f} \alpha^{\prime}<\alpha . \exists_{c f} \beta^{\prime}<\alpha^{\prime+} .\left(a_{\alpha^{\prime}, \beta^{\prime}, i^{\prime}} \mid i^{\prime} \in \omega\right) \text { subsumes }\left(\left(\alpha^{\prime}, \beta^{\prime}, i^{\prime}\right) \mid i^{\prime} \in \omega\right) .
$$

Pick $\alpha_{0}$ such that $\kappa \leq \alpha_{0}<\alpha$ and the set

$$
C:=\left\{\beta^{\prime}<\alpha_{0}^{+} \mid\left(a_{\alpha_{0}, \beta^{\prime}, i^{\prime}} \mid i^{\prime} \in \omega\right) \text { subsumes }\left(\left(\alpha_{0}, \beta^{\prime}, i^{\prime}\right) \mid i^{\prime} \in \omega\right)\right\}
$$

is cofinal in $\alpha_{0}^{+}$and hence has size $\alpha_{0}^{+}>\alpha_{0} \geq \kappa$. Applying Lemma 5.3, for each $\beta^{\prime} \in C$ we can pick $j\left(\beta^{\prime}\right)$ and $k\left(\beta^{\prime}\right) \neq k^{\prime}\left(\beta^{\prime}\right)$ such that

$$
\left(\alpha_{0}^{\prime}, \beta^{\prime}, k\left(\beta^{\prime}\right)\right) \in a_{\alpha_{0}^{\prime}, \beta^{\prime}, j\left(\beta^{\prime}\right)}, \quad\left(\alpha_{0}^{\prime}, \beta^{\prime}, k^{\prime}\left(\beta^{\prime}\right)\right) \in a_{\alpha_{0}^{\prime}, \beta^{\prime}, j\left(\beta^{\prime}\right)}
$$

and therefore $\left|a_{\alpha_{0}^{\prime}, \beta^{\prime}, j\left(\beta^{\prime}\right)}\right|>1$. We thus obtained a subset of $\langle x\rangle_{\Sigma_{\mathcal{M}}^{\kappa}}$ of cardinality strictly greater than $\kappa$ whose elements are non-singleton sets. However, $\langle x\rangle_{\Sigma_{\kappa}}$ can have at most $\kappa$ non-singleton elements, for $|X|=\kappa$ and application of functions from $\Sigma_{\kappa}$ can only introduce either singletons or the empty set. Contradiction.

\footnotetext{
${ }^{1}$ Recall that a subset $C$ of $\alpha$ is cofinal in $\alpha$ if and only if $\forall \beta<\alpha . \exists \gamma \in C . \beta \leq \gamma$
} 
We now go on to show that the $M_{\delta}$ disprove existence of the tensor $\mathcal{M} \otimes \mathcal{P}_{\omega}$.

Lemma 5.5. Let $X$ be a set, $\left(x_{i} \mid i \in \omega\right)$ a sequence of elements of $X$, and $\left(a_{i} \mid i \in \omega\right)$ and $\left(b_{i} \mid i \in \omega\right)$ sequences of finite subsets of $X$. Then $\left(a_{i} \cup b_{i} \mid i \in \omega\right)$ subsumes $\left(x_{i} \mid i \in \omega\right)$ if and only if at least one of $\left(a_{i} \mid i \in \omega\right)$ and $\left(b_{i} \mid i \in \omega\right)$ does.

Proof. The 'if' direction is immediate. For the other direction, we employ Ramsey's theorem, which states that for any coloring of the set of pairs of natural numbers with two colours there is an infinite set of natural numbers which is monochromatic in the sense that all the pairs of numbers from that set are the same colour. Without loss of generality, the set $M$ witnessing that $\left(a_{i} \cup b_{i} \mid i \in \omega\right)$ subsumes $\left(x_{i} \mid i \in \omega\right)$ is the whole of $\omega$. Now for any $i<j<\omega$, we colour the pair $\{i, j\}$ red if $x_{i} \in a_{j}$ and blue otherwise. In this way we colour all 2-element subsets of $\omega$. By Ramsey's theorem, we can find a subset $M^{\prime}$ of $\omega$ such that all pairs from $M^{\prime}$ were given the same colour. If they were all coloured blue, then for each $i$ and $j$ in $M^{\prime}$ with $i<j$ we have $x_{i} \in\left(a_{j} \cup b_{j}\right) \backslash a_{j} \subseteq b_{j}$, so $\left(b_{i} \mid i \in \omega\right)$ subsumes $\left(x_{i} \mid i \in \omega\right)$. A similar argument shows that if all pairs from $M^{\prime}$ are coloured red then $\left(a_{i} \mid i \in \omega\right)$ subsumes $\left(x_{i} \mid i \in \omega\right)$.

Lemma 5.6. In $M_{\delta}$, each map $f_{\alpha, \beta, i}$ and the constant $c$ commute with the bounded semilattice structure (i.e. the $\mathcal{P}_{\omega}$-algebra structure on $\left.M_{\delta}\right)$; in other words, any $M_{\delta}$ is a small $\left(\mathcal{M}, \mathcal{P}_{\omega}\right)$-tensor algebra.

Proof. For $f_{\alpha, \beta, i}$, the case $\alpha \geq \delta$ is trivial; so assume $\alpha<\delta$. Since $(\varnothing \mid i \in \omega)$ never subsumes anything, we have $f_{\alpha, \beta, i}\left(\varnothing \mid\left(\alpha^{\prime}, \beta^{\prime}, i^{\prime}\right) \in B_{\alpha}\right)=\varnothing$, so $f_{\alpha, \beta, i}$ commutes with $\perp$. Because of the form of $f_{\alpha, \beta, i}$, to check that it commutes with $\vee$ it is enough to check that for any two families $\left(a_{\alpha^{\prime}, \beta^{\prime}, i^{\prime}} \mid\left(\alpha^{\prime}, \beta^{\prime}, i^{\prime}\right) \in B_{\alpha}\right)$ and $\left(b_{\alpha^{\prime}, \beta^{\prime}, i^{\prime}} \mid\left(\alpha^{\prime}, \beta^{\prime}, i^{\prime}\right) \in B_{\alpha}\right)$, the following two conditions are equivalent

- $\exists_{c f} \alpha^{\prime}<\alpha . \exists_{c f} \beta^{\prime}<\alpha^{\prime+} .\left(a_{\alpha^{\prime}, \beta^{\prime}, i^{\prime}} \cup b_{\alpha^{\prime}, \beta^{\prime}, i} \mid i^{\prime} \in \omega\right)$ subsumes $\left(\left(\alpha^{\prime}, \beta^{\prime}, i^{\prime}\right) \mid i^{\prime} \in \omega\right)$

- either $\exists_{c f} \alpha^{\prime}<\alpha . \exists_{c f} \beta^{\prime}<\alpha^{\prime+}$. $\left(a_{\alpha^{\prime}, \beta^{\prime}, i^{\prime}} \mid i^{\prime} \in \omega\right)$ subsumes $\left(\left(\alpha^{\prime}, \beta^{\prime}, i^{\prime}\right) \mid i^{\prime} \in \omega\right)$

or $\exists_{c f} \alpha^{\prime}<\alpha . \exists_{c f} \beta^{\prime}<\alpha^{\prime+}$. $\left(b_{\alpha^{\prime}, \beta^{\prime}, i^{\prime}} \mid i^{\prime} \in \omega\right)$ subsumes $\left(\left(\alpha^{\prime}, \beta^{\prime}, i^{\prime}\right) \mid i^{\prime} \in \omega\right)$.

This equivalence is immediate from Lemma 5.5 and the fact that a union of two subsets of an ordinal $\alpha$ is cofinal in $\alpha$ if and only if at least one of those two subsets is cofinal in $\alpha$.

Finally, $c=\perp=\varnothing$ and hence $c \vee c=c$, i.e. $c$ also commutes with the semilattice structure.

Lemma 5.7. Any $M_{\kappa}$ is $\aleph_{0}$-reachable, specifically generated from the countable set $X=$ $\left\{\{b\} \mid b \in B_{1}\right\}$ under the operations of $\Sigma$ and the bounded semilattice structure.

Proof. Let $A^{\prime}$ be the subset of $M_{\kappa}$ generated in this way. It suffices to prove that for each $\alpha \leq \kappa$ we have $\left\{\{(\alpha, \beta, i)\} \mid \beta<\alpha^{+} \wedge i<\omega\right\} \subseteq A^{\prime}$. If $\alpha=0$ this is true by definition. Otherwise, this is true by induction on $\alpha$, using the equation

$$
\{(\alpha, \beta, i)\}=f_{\alpha, \beta, i}\left(\left\{\left(\alpha^{\prime}, \beta^{\prime}, j\right) \mid j<i^{\prime}\right\} \mid\left(\alpha^{\prime}, \beta^{\prime}, i^{\prime}\right) \in B_{\alpha}\right) .
$$

We have now shown that $B \subseteq A^{\prime}$, and $A \subseteq A^{\prime}$ is immediate.

By 3.11, we obtain

Theorem 5.8. The tensor of $\mathcal{M}$ and $\mathcal{P}_{\omega}$ does not exist (not even as a spurious tensor).

Remark 5.9. By Proposition 3.9, the above result implies that every monad that is induced by a theory that has one binary operation and at most one constant and whose equations are implied by those of $\mathcal{P}_{\omega}$ (associativity, commutativity, and idempotence of the binary 
operation, neutrality of the constant if any) fails to be tensorable. In particular, finite nonempty powerset and both the full and the non-empty versions of the list monad and and the finite multiset monad, respectively, fail to be tensorable.

\section{A Well-Order Monad That Fails to Be Tensorable}

As announced above, we now present a second example of two monads whose tensor fails to exist, originally published in [8]. It involves a well-order monad $\mathcal{W}$, where $\mathcal{W}(X)$ consists of all well-orderings on non-empty subsets of $X$, plus an error element; the other partner is ranked, a free monad over two binary operations. The construction follows the same pattern as in the preceding section: first we introduce a large theory of $\mathcal{T}_{\mathcal{W}}$-algebras, then we show that this theory yields a monad $\mathcal{W}$, and finally we prove that the tensor product $\mathcal{W}$ with the ranked partner does not exist using Corollary 3.11.

Definition 6.1. The theory $\mathcal{T}_{\mathcal{W}}$ of strict non-empty well-orders has a signature consisting of a constant $\perp$ and a family of operation symbols $\iota_{\kappa}$ of arity $\kappa$, indexed over all positive ordinals $\kappa$. It imposes the following equations.

(1) Strictness: $\iota_{\kappa}\left(w_{\alpha} \mid \alpha<\kappa\right)=\perp$ whenever $w_{\alpha}=\perp$ for some $\alpha<\kappa$.

(2) Non-repetitiveness: $\iota_{\kappa}\left(w_{\alpha} \mid \alpha<\kappa\right)=\perp$ whenever $w_{\alpha_{1}}=w_{\alpha_{2}}$ for some $\alpha_{1}<\alpha_{2}<\kappa$.

(3) Associativity: For every small-ordinal-indexed family $\left(\kappa_{\mu}\right)_{\mu<\nu}$ of ordinals $\kappa_{\mu}>0$,

$$
\iota_{\kappa}\left(w_{\mu, \alpha} \mid \mu<\nu, \alpha<\kappa_{\mu}\right)=\iota_{\nu}\left(\iota_{\kappa_{\mu}}\left(w_{\mu, \alpha} \mid \alpha<\kappa_{\mu}\right) \mid \mu<\nu\right)
$$

where on the left hand side $\kappa=\sum_{\mu<\nu} \kappa_{\mu}$ is regarded as having elements $\langle\mu, \alpha\rangle$ with $\mu<\nu$ and $\alpha<\kappa_{\mu}$.

We regard an ordinal $\kappa$ as the set of all ordinals $\alpha<\kappa$ unless we explicitly specify otherwise, as in the associativity law above where we use a more convenient isomorphic representation of ordinal sums. Even though in the above formulations of strictness and non-repetitiveness we employ the word 'whenever', they may nevertheless be interpreted as sets of equational axioms.

Now consider a small $\mathcal{T}_{\mathcal{W}}$-algebra $X$. By non-repetitiveness, for every $\kappa$ whose cardinality exceeds $|X|, \iota_{\kappa}$ is identically $\perp$, which means that the set of nontrivial operations in the structure of any particular $\mathcal{T}_{\mathcal{W}}$ is small. A homomorphism of two $\mathcal{T}_{\mathcal{W}}$-algebras $\left(X, \iota_{\kappa}\right)$ and $\left(Y, \iota_{\kappa}\right)$ is a map $f: X \rightarrow Y$ that commutes with the operations, i.e.

$$
f\left(\iota_{\kappa}\left(w_{\alpha} \mid \alpha<\kappa\right)\right)=\iota_{\kappa}\left(f\left(w_{\alpha}\right) \mid \alpha<\kappa\right) \text { for } w \in X^{\kappa} .
$$

Lemma and Definition 6.2. The theory $\mathcal{T}_{\mathcal{W}}$ has small free algebras. The induced monad $\mathcal{W}$, the strict non-empty well-order monad, maps a small set $X$ to the set

$$
\mathcal{W} X=\{(Y, \rho) \mid \varnothing \neq Y \subseteq X, \rho \text { a well-order on } Y\} \cup\{\perp\} \text {. }
$$

Its unit maps $x \in X$ to unique well-order on $\{x\}$, and its multiplication concatenates wellorders in case all its arguments are well-orders whose carriers are pairwise disjoint (so that the result is again a well-order), and otherwise returns $\perp$.

One may alternatively think of the strict non-empty well-order monad as a monad of infinite non-repetitive non-empty lists, with $\perp$ playing the role of an error element that is thrown in case of repetitions arising by concatenation, and that is propagated through concatenation by the strictness law. 
Proof. It is easy to see that the elements of $\mathcal{W}(X)$ serve as unique normal forms in $F_{\mathcal{T}_{\mathcal{W}}}(X)$.

The second monad for our example is very simple, and has finite rank: Let $\Sigma_{2,2}^{\star}$ be the free algebra monad for the empty theory in the signature $\Sigma_{2,2}$ consisting of just 2 binary operations.

Lemma 6.3. For every infinite cardinal $\kappa$, there exists a 2 -reachable small $\left(\mathcal{W}, \Sigma_{2,2}^{\star}\right)$-tensor algebra $W_{\kappa}$ such that $\left|W_{\kappa}\right|>\kappa$.

Proof. The domain of $W_{\kappa}$ is the union $\{\perp, 0,1\} \cup U_{\kappa}^{0} \cup U_{\kappa}^{1} \cup L_{\kappa}$ where the $U_{\kappa}^{i}$ and $L_{\kappa}$ are sets of terms defined by infinitary mutual recursion according to the the rules

$$
\frac{t \in W_{\kappa}-\{0\}}{\langle i, 0, t\rangle \in U_{\kappa}^{i}}
$$

where $i \in\{0,1\}$, and

$$
\frac{t: \nu \hookrightarrow U_{\kappa}^{0} \cup U_{\kappa}^{1} \quad \forall \mu \cdot \mu+1<\nu \Longrightarrow\left(t(\mu) \in U_{\kappa}^{0} \Longleftrightarrow t(\mu+1) \in U_{\kappa}^{1}\right)}{t \in L_{\kappa}}
$$

where $\nu$ is an ordinal such that $1<|\nu| \leq \kappa$ and $\hookrightarrow$ is read as $t$ being injective (not a subset inclusion). Notice that $U_{\kappa}^{0} \cap U_{\kappa}^{1}=\varnothing$, so the second premise says that $t(\mu)$ alternates between $U_{\kappa}^{0}$ and $U_{\kappa}^{1}$. Let us define a length map \# from $W_{\kappa}$ to ordinals as follows: we put

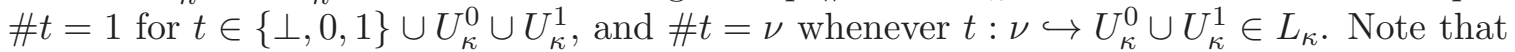
this implies \#t>1 iff $t \in L_{\kappa}$.

To give a $\Sigma_{2,2}^{\star}$-algebra structure over $W_{\kappa}$ is the same as to define two binary maps $u_{0}, u_{1}: W_{\kappa} \times W_{\kappa} \rightarrow W_{\kappa}$. For $i=0,1$ we put by definition

- $u_{i}(t, t)=t$ if $t \in\{0,1\}$;

- $u_{i}(0, t)=\langle i, 0, t\rangle \in U_{\kappa}^{i}$ whenever $t \in\{1\} \cup L_{\kappa}$;

- $u_{i}(s, t)=\perp$ in the remaining cases.

We now define a $\mathcal{T}_{\mathcal{W}}$-algebra structure on $W_{\kappa}$. We interpret $\perp$ by $\perp$, and $\iota_{1}$ by id. For $\nu>1$ and $t \in\left(W_{\kappa}\right)^{\nu}$ we define $\iota_{\nu}(t)$ by the clauses

- $\iota_{\nu}(t)=s$, provided the map $s: \zeta \rightarrow W_{\kappa}$ on $\zeta=\sum_{\mu<\nu} \# t(\mu)$ defined as follows is in $L_{\kappa}$ : We regard $\zeta$ as consisting of pairs $\langle\mu, \kappa\rangle$ where $\mu<\nu$ and $\kappa<\# t(\mu)$. For every such $\langle\mu, \kappa\rangle$, put $s\langle\mu, \kappa\rangle=t(\mu)(\kappa)$ if $t(\mu) \in L_{\kappa}$, and $s\langle\mu, \kappa\rangle=t(\mu)$ otherwise (in which case necessarily $\kappa=0)$.

- $\iota_{\nu}(t)=\perp$ otherwise.

It is then clear by construction that $W_{\kappa}$ is 2 -reachable (it is generated by 0 and 1 ), as the rules defining $L_{\kappa}$ and the $U_{\kappa}^{i}$ just amount to closure under the $u_{i}$ and $\iota_{\nu}$ as defined above. Next, we have to check that $W_{\kappa}$ is really a $\mathcal{T}_{\mathcal{W}}$-algebra. By definition, for every $t$, $\iota_{\nu}(t) \in L_{\kappa} \cup\{\perp\}$, hence the conditions (1) and (2) of Definition 6.1 are ensured automatically. Condition (3) is less trivial, but still routine. Finally we need to verify the tensor law. In the case at hand it amounts to proving the equation

$$
u_{i}\left(\iota_{\nu}(t), \iota_{\nu}(s)\right)=\iota_{\nu}\left(\lambda \mu<\nu \cdot u_{i}(t(\mu), s(\mu))\right)
$$

for every $s, t \in W_{\kappa}, i=0,1$. It is immediate by definition that both sides of this equation equal $\perp$ unless $\nu=1$. In the latter case the equation also follows since, by definition, $\iota_{1}=\mathrm{id}$. 
Finally, we show that $\left|W_{\kappa}\right|>\kappa$. In order to derive a contradiction, assume that $\left|W_{\kappa}\right| \leq \kappa$ and let $\varsigma$ be an ordinal number such that $\left|W_{\kappa}\right|=|\varsigma|$. Let $\rho$ be a bijection $\varsigma \rightarrow W_{\kappa}-\{0\}$. Since $|\varsigma| \leq \kappa$ and hence $|\varsigma \cdot 2| \leq \kappa$ (since $\kappa$ is infinite), we can form an element $t_{\rho}: \varsigma \cdot 2 \hookrightarrow U_{\kappa}^{0} \cup U_{\kappa}^{1}$ of $W_{\kappa}$ by putting $t_{\rho}\left(\varsigma^{\prime}, i\right)=\left\langle i, 0, \rho\left(\varsigma^{\prime}\right)\right\rangle$ for $\varsigma^{\prime}<\varsigma, i=0,1$. By varying $\rho$, we can produce as many such elements as there are isomorphisms from $\varsigma$ to $W_{\kappa}-\{0\}$, i.e. strictly more than $|\varsigma|=\left|W_{\kappa}\right|$, contradiction.

By Corollary 3.11 we obtain

Theorem 6.4. The tensor of the strict non-empty well-order monad $\mathcal{W}$ and $\Sigma_{2,2}^{\star}$ does not exist, even as a spurious tensor.

\section{Conclusion}

Tensors of theories, or monads, capture algebras of one theory in the category of algebras of the other. For unranked monads, equivalently large theories with small free algebras, existence of tensors is not self-understood; we call a theory or monad tensorable if its tensors with all other theories, or monads, respectively, exist. We have given two counterexamples to tensorability of monads:

- the tensor of the finite powerset monad with a certain somewhat complex unranked monad fails to exist;

- the tensor of the strict nonempty well-order monad and a simple finitary monad, generated by two binary operations and no equations, fails to exist.

We have thus settled in the negative the long-standing open question of universal existence of tensors of monads on Set [17], which has recently reemerged in the perspective of work on algebraic effects [12, 11]. The negative answer as such is in accordance with expectations, but the actual counterexamples are rather different from what was previously suspected.

In addition to our negative results, we have established a positive result stating that all bounded powerset monads - except finite powerset - are (genuinely) tensorable.

Our main motivation for the study of tensors as such is to develop a monadic framework for non-interference of side-effects, noting that the tensor law precisely amounts to orthogonality of the component monads; these ideas will be further developed in future research. Another topic of further interest is the investigation of tensors over base categories other than Set, for example the category of $\omega$-complete partial orders.

Acknowledgements. We wish to thank various contributors to the categories mailing list, in particular Peter Johnstone, for useful insights communicated via the list, and the anonymous referees of [8] for valuable pointers to the literature.

\section{REFERENCES}

[1] J. Adámek, N. Bowler, P. B. Levy, and S. Milius. Coproducts of monads on set. In N. Dershowitz, ed., Logic in Computer Science, LICS 2012, pp. 45-54. IEEE, 2012.

[2] M. Barr and C. Wells. Toposes, Triples and Theories, vol. 278 of Grundlehren der mathematischen Wissenschaften. Springer, 1985.

[3] P. Cenciarelli and E. Moggi. A syntactic approach to modularity in denotational semantics. In Category Theory and Computer Science, CTCS 1993, 1993.

[4] M. Droste, W. Kuich, and H. Vogler, eds. Handbook of Weighted Automata. Springer, 2009. 
[5] E. Dubuc. Kan Extensions in Enriched Category Theory, vol. 145. Springer, 1970.

[6] U. Felgner. Comparison of the axioms of local and universal choice. Fundamenta Mathematicae, 71:4362, 1971.

[7] P. Freyd. The theory of functors and models. In Theory of Models - Proceedings of the 1963 International Symposium at Berkeley, pp. 107-120. North Holland, 1966.

[8] S. Goncharov and L. Schröder. A counterexample to tensorability of effects. In A. Corradini and B. Klin, eds., Algebra and Coalgebra in Computer Science, CALCO 2011, Lect. Notes Comput. Sci., pp. 208-211. Springer, 2011.

[9] S. Goncharov and L. Schröder. Powermonads and tensors of unranked effects. In M. Grohe, ed., Logic in Computer Science, LICS 2011, pp. 227-236. IEEE Computer Society, 2011.

[10] S. Goncharov, L. Schröder, and T. Mossakowski. Kleene monads: handling iteration in a framework of generic effects. In A. Kurz and A. Tarlecki, eds., Algebra and Coalgebra in Computer Science, CALCO 2009, vol. 5728 of Lect. Notes Comput. Sci., pp. 18-33. Springer, 2009.

[11] M. Hyland, P. B. Levy, G. Plotkin, and J. Power. Combining algebraic effects with continuations. Theoret. Comput. Sci., 375(1-3):20 - 40, 2007. Festschrift for John C. Reynolds's 70th birthday.

[12] M. Hyland, G. Plotkin, and J. Power. Combining effects: Sum and tensor. Theoret. Comput. Sci., 357:70-99, 2006.

[13] M. Hyland and J. Power. The category theoretic understanding of universal algebra: Lawvere theories and monads. In Computation, Meaning, and Logic: Articles dedicated to Gordon Plotkin, vol. 172 of Electron. Notes Theoret. Comput. Sci., pp. 437-458. Elsevier, 2007.

[14] G. M. Kelly. A unified treatment of transfinite constructions for free algebras, free monoids, colimits, associated sheaves, and so on. Bull. Austral. Math. Soc., 22:1-84, 1980.

[15] F. Linton. Some aspects of equational categories. In Conference on Categorical Algebra, La Jolla, pp. 84-94. Springer, 1966.

[16] C. Lüth and N. Ghani. Composing monads using coproducts. In International Conference on Functional Programming, ICFP 2002, vol. 37(9) of SIGPLAN Not., pp. 133-144. ACM, 2002.

[17] E. Manes. A triple theoretic construction of compact algebras. In Seminar on Triples and Categorical Homology Theory, vol. 80 of Lect. Notes Math., pp. 91-118. Springer, 1969.

[18] E. Moggi. Notions of computation and monads. Inf. Comput., 93:55-92, 1991.

[19] E. Moggi. A semantics for evaluation logic. Fund. Inform., 22:117-152, 1995.

[20] A. P. Morse. A theory of sets. Academic Press, 1965.

[21] S. Peyton-Jones, ed. Haskell 98 Language and Libraries - The Revised Report. Cambridge University Press, 2003. Also: J. Funct. Prog. 13 (2003).

[22] G. Plotkin and J. Power. Notions of computation determine monads. In M. Nielsen and U. Engberg, eds., Foundations of Software Science and Computation Structures, FOSSACS 2002, vol. 2303 of Lect. Notes Comput. Sci., pp. 342-356. Springer, 2002.

[23] J. Power and O. Shkaravska. From comodels to coalgebras: State and arrays. In J. Adámek and S. Milius, eds., Coalgebraic Methods in Computer Science, CMCS 2004, vol. 106 of Electron. Notes Theoret. Comput. Sci., pp. 297-314, 2004.

[24] L. Schröder and T. Mossakowski. Generic exception handling and the Java monad. In C. Rattray, S. Maharaj, and C. Shankland, eds., Algebraic Methodology and Software Technology, AMAST 2004, vol. 3116 of Lect. Notes Comput. Sci., pp. 443-459. Springer, 2004.

[25] D. Scott. Definitions by abstraction in axiomatic set theory. Bull. AMS, 61(442):8, 1955.

[26] P. Wadler. How to declare an imperative. ACM Comput. Surveys, 29:240-263, 1997. 